PROCEEDINGS OF THE

AMERICAN MATHEMATICAL SOCIETY

Volume 131, Number 1, Pages 29-33

S 0002-9939(02)06516-4

Article electronically published on July 15, 2002

\title{
SEMIPRIME CROSSED PRODUCTS OVER COPOINTED HOPF ALGEBRAS
}

\author{
DECLAN QUINN AND ŞERBAN RAIANU
}

(Communicated by Matin Lorenz)

\begin{abstract}
We prove a result on the transfer of essentiality of extensions of modules over subnormalizing extensions of rings, then apply it to look at the semiprimeness of Hopf-Galois extensions, in particular that of crossed products.
\end{abstract}

The following is an important open question in the theory of Hopf algebra actions (see 12, Question 7.4.9, p.121]):

If $H$ is a finite-dimensional and semisimple Hopf algebra over the field $k$, and $A$ is semiprime, is any crossed product $A \#{ }_{\sigma} H$ semiprime?

This possible extension of the Maschke-type theorem for crossed products [2], was inspired by the fact that it holds in the following cases:

1. when $H=k G$ ( $G$ is a finite group, and $|G|^{-1} \in k$ ) (this is the FisherMontgomery theorem, 9]), or

2. when $H=k G^{*}[6]$.

The first case was extended in [3] to the case where $H$ is semisimple, pointed and cocommutative. The aim of the present note is to try to extend the second case, exploiting the fact that the dual Hopf algebra $H^{*}$ is pointed in this case. The result of this effort is Corollary [5] The technique that we use involves the socalled essential form of Maschke's theorem. This approach was first used to provide another proof for the Fisher-Montgomery theorem in [15]. Various partial answers to the above question were also given in 1], [17, 13.

Throughout, $H$ will denote a finite-dimensional Hopf algebra over the field $k$. For all unexplained notation or definitions, the reader is referred to [12].

Recall that if $R$ is a subring of $S$, the extension $R \subset S$ is called a subnormalizing (or triangular) extension if there exist elements $x_{1}, x_{2}, x_{3}, \ldots \in S$ such that $S=$ $\sum_{i=1}^{\infty} R x_{i}$ and for any $j$ we have $\sum_{i=1}^{j} R x_{i}=\sum_{i=1}^{j} x_{i} R$. A subnormalizing extension is called finite if the sequence $x_{1}, x_{2}, x_{3}, \ldots \in S$ is finite (see [18] or [11]). Our first result is

Theorem 1. Let $R \subset S$ be a subnormalizing extension of rings, such that the elements $x_{1}, x_{2}, x_{3}, \ldots \in S$ from the definition form a basis for $S$ as a left and $a$ right $R$-module. If $M \subset N$ is an essential extension of left $R$-modules, and $N$ is nonsingular, then $S \otimes_{R} M \subset S \otimes_{R} N$ is an essential extension of $R$-modules.

Received by the editors May 23, 2001 and, in revised form, August 8, 2001.

2000 Mathematics Subject Classification. Primary 16W30.

The second author is on leave from University of Bucharest, Faculty of Mathematics. 
Proof. Suppose the extension $S \otimes_{R} M \subset S \otimes_{R} N$ is not essential. We say $w \in$ $S \otimes_{R} N, w \neq 0$, is a nasty element if any $R$-multiple of it is either 0 or not in $S \otimes_{R} M$. It is clear that a nonzero $R$-multiple of a nasty element is again nasty. Among all nasty elements choose $x_{1} \otimes n_{1}+\ldots+x_{t} \otimes n_{t} \in S \otimes_{R} N$ with $j$ minimal subject to $n_{j} \notin M, n_{j+1} \in M, \ldots, n_{t} \in M$.

For any $i$ there exists an automorphism $\sigma_{i}: R \longrightarrow R$ such that $a x_{i}=x_{i} \sigma_{i}(a)+$ lower terms for all $a \in R$. If $a \in R$, then

$$
a w=\ldots+x_{j} \otimes\left(\sigma_{j}(a) n_{j}+n^{\prime}\right)+x_{j+1} \otimes n_{j+1}^{\prime}+\ldots+x_{t} \otimes n_{t}^{\prime}
$$

where $n^{\prime}, n_{j+1}^{\prime}, \ldots n_{t}^{\prime} \in M$, and by the minimality of $j$ we have that

$$
\operatorname{l.ann}_{R}(w)=\sigma_{j}^{-1}\left(\operatorname{l.ann}_{R}\left(\widehat{n_{j}}\right)\right),
$$

where $\widehat{n_{j}}=n_{j}+M \in M / N$. But $\operatorname{lann}_{R}\left(\widehat{n_{j}}\right)$ is an essential ideal of $R$, because the extension $M \subset N$ is essential. Thus $\operatorname{lann}_{R}(w)$ is also an essential ideal.

Now, for $a \in \operatorname{l.ann}_{R}(w)$ we have

$$
0=a w=x_{1} \otimes n_{1}^{\prime}+\ldots+x_{t-1} \otimes n_{t-1}^{\prime}+x_{t} \otimes \sigma_{t}(a) n_{t},
$$

so $\operatorname{lann}_{R}(w) \subseteq \sigma_{t}^{-1}\left(1 \cdot \operatorname{ann}_{R}\left(n_{t}\right)\right)$, and hence $1 \cdot \operatorname{ann}_{R}\left(n_{t}\right)$ is also an essential ideal, contradicting the fact that $N$ is nonsingular.

This implies that $S \otimes_{R} M$ has no nasty elements and the result follows.

The next lemma is an application of the Taft-Wilson theorem, and was proved in [7] (see also [8, Exercise 7.7.9, p.338]).

Lemma 2. Let $H$ be a finite-dimensional pointed Hopf algebra acting on the algebra $A$. Then $A \# H$ is a finite subnormalizing extension of $A$, and the elements $x_{1}, \ldots, x_{m} \in A \# H$ may be chosen to form a basis of $H \subset A \# H$.

We are now in a position to state and prove our main result.

Theorem 3. Let $H$ be a finite-dimensional pointed Hopf algebra, and $A$ a left $H$ module algebra with subalgebra of invariants $A^{H}$, such that $A / A^{H}$ is a faithfully flat right $H^{*}$-Galois extension. Then the following hold:

a) If $M \subset_{A} N$ is an essential extension of left $A$-modules, and $N$ is left nonsingular, then $N$ is an essential extension of $M$ as left $A^{H}$-modules.

b) If $A$ is $H$-semiprime and $A$ is a left nonsingular ring, then $A$ is semiprime.

c) If $A^{H}$ is semiprime and $A$ is a left nonsingular ring, then $A$ is semiprime.

Proof. a) The proof uses the duality approach of [6] as in [16] and [4], in the form for Hopf algebra actions which was also used in [7]: since the induced functor $A \# H \otimes_{A}-: A-\bmod \longrightarrow(A \# H) \# H^{*}-\bmod$ is an equivalence, it follows that

$$
A \# H \otimes_{A} M \subset A \# H \otimes_{A} N
$$

is an essential extension of left $(A \# H) \# H^{*}$-modules.

By Lemma 2, we can apply Theorem 1 for the subnormalizing extension $A \subset$ $A \# H$ to obtain that (1) is an essential extension of left $A$-modules, and so it is also an essential extension of left $A \# H$-modules.

Now we have the functorial isomorphisms of left $A \# H$-modules $A \# H \otimes_{A} M \simeq$ $A \otimes_{A^{H}} M$, and $A \# H \otimes_{A} N \simeq A \otimes_{A^{H}} N$, so $A \otimes_{A^{H}} M \subset A \otimes_{A^{H}} N$ is an essential extension of left $A \# H$-modules. But the induced functor $A \otimes_{A^{H}}-: A^{H}-\bmod \longrightarrow$ $A \# H-\bmod$ is also an equivalence, since $A / A^{H}$ is a faithfully flat Galois extension, and therefore we have that $M \subset_{A^{H}} N$ is an essential extension. 
b) The proof is similar to the proof of the Fisher-Montgomery theorem, as given in [15], but we sketch it for the convenience of the reader. Let $0 \neq N$ be an ideal of $A$ such that $N^{2}=0$. Then $I=\mathrm{r} \cdot \operatorname{ann}_{A}(N)$ is an essential left ideal of $A$. By a), we get that $I$ is an essential $A^{H}$-submodule of $A$, so $I^{H}=I \cap A^{H}$ is an essential left ideal of $A^{H}$. Let

$$
J=(I: H)=\{a \in I \mid h \cdot a \in I \quad \forall h \in H\} .
$$

Since $I^{H} \subseteq J \subseteq I$, it follows that $J^{H}=I^{H}$, and since the extension is Galois, we get that $J=I^{H} A$, by [12, Corollary 8.3.10, p.138]. But now

$$
\operatorname{l.ann}_{A}(J)=1 \cdot \operatorname{ann}_{A}\left(I^{H}\right) \supseteq \operatorname{lann}_{A}(I) \supseteq N \neq 0 .
$$

Thus $L=\operatorname{lann}_{A}(J)$ is a nonzero $H$-stable ideal of $A$, which is again generated (as a left ideal) by its intersection with $A^{H}$. Hence we have that $L^{H} \cap I^{H} \neq 0$. Finally, $J \cap L$ is a nonzero $H$-stable ideal of $A$ with $(J \cap L)^{2}=0$, a contradiction to the fact that $A$ is $H$-semiprime.

c) follows from b), because $H$-stable ideals of $A$ are generated by their intersection with $A^{H}$.

The following is a corollary of the proof of Theorem 3 and is presumably known.

Corollary 4. If $G$ is a finite group acting as automorphisms on the $k$-algebra $R$, $R^{G}$ denotes the subalgebra of invariants, and $R / R^{G}$ is a Galois extension having an element of trace 1 , then the following hold:

a) If $M \subset_{R} N$ is an essential extension of left $R$-modules, then $M \subset N$ is also an essential extension as left $R^{G}$-modules.

b) If $R^{G}$ is semiprime, then $R$ is semiprime.

Proof. As in the proof of part a) of Theorem 3. we want to prove that

$$
R * k G \otimes_{R} M \subset R * k G \otimes_{R} N
$$

is an essential extension of $R * k G$-modules. But since this is an essential extension of left $(R * k G) \# k G^{*}$-modules, i.e. a gr-essential extension of graded $R * k G$-modules, the claim follows easily from [14, I.2.8, p. 9]. The rest of the proof is the same.

A direct consequence of Theorem 3 is the following.

Corollary 5. Let $H$ be a finite-dimensional Hopf algebra such that $H^{*}$ is pointed, and $A \#_{\sigma} H$ a crossed product with invertible cocycle $\sigma$. If $A$ is $H$-semiprime and $A \#{ }_{\sigma} H$ is a left nonsingular ring, then $A \#{ }_{\sigma} H$ is also semiprime.

Proof. Denote the left weak action of $H$ on $A$ by $h \cdot a$ for $a \in A$ and $h \in H$.

We remark first that if $A$ is $H$-semiprime, then $A \#{ }_{\sigma} H$ is $H^{*}$-semiprime. Indeed, if $N$ is a nonzero $H^{*}$-stable ideal of $A \#{ }_{\sigma} H$ with $N^{2}=0$, then $N \cap A$ generates $N$ as a left ideal (again by [12 Corollary 8.3.10, p.138]), so $N \cap A \neq 0$, and clearly $(N \cap A)^{2}=0$. Moreover, $N \cap A$ is an $H$-stable ideal of $A$, since for $n \in N \cap A$ and $h \in H$, we have by [12, 7.2.3 and 7.2.7] that

$$
h \cdot n=\sum\left(1 \# h_{1}\right)(n \# 1)\left(\sigma^{-1}\left(S\left(h_{3}\right), h_{4}\right) \# S\left(h_{2}\right)\right) .
$$

This provides the desired contradiction.

Now apply part b) of Theorem 3 to finish the proof. 
Remark 6 . The relationship between the above Corollary and the question mentioned in the beginning of this note is as follows: the condition " $A \#_{\sigma} H$ is nonsingular" is needed as a replacement for the condition " $H$ is semisimple" (the latter would imply that $H=k G$ for some finite group $G$, and this would bring us back to the graded case). This can be seen by taking $A$ to be trivial. Then $H$ nonsingular is equivalent to $H$ semisimple, or $H$ semiprime, because $H$ is Frobenius (see [10. 13.2 , p.362]).

A simple example where $A \# H$ is nonsingular (in fact, even simple) without $H$ being semisimple is as follows: let $\mathbf{F}_{2}$ be the field with two elements, $E=\mathbf{F}_{2}(X)$ the field of rational fractions in the indeterminate $X$, and let $k$ be the subfield $\mathbf{F}_{2}\left(X^{2}\right)$. Let $\delta=\frac{d}{d X} \in \operatorname{Der}(E)$, and denote by $L$ the 1-dimensional Lie algebra generated by $\delta$. Consider the action of $L$ on $E$ as derivations. Then the invariants of this action are just $k$ itself, and the Hopf algebra is $H=U(L) \simeq H^{*}$. The extension is Galois. This follows immediately from a result in [5] which may also be found in [12, 8.3.7, p. 137], or it can be seen directly after a short computation. Now, since the extension is Galois, the smash product $E \# H$ is isomorphic to $E_{n d}(E)$, which is a simple ring.

\section{ACKNOWLEDGMENT}

We thank Susan Montgomery for helpful conversations, and the referee for suggesting improvements in the exposition.

\section{REFERENCES}

[1] J. Bergen, S. Montgomery, Ideals and quotients in crossed products of Hopf Algebras, J. Algebra 152 (1992), 374-439. MR 94a:16054

[2] R.J. Blattner, S. Montgomery, Crossed products and Galois extension of Hopf algebras, Pacific J. Math. 137 (1989), 37-54. MR 90a:16007

[3] W. Chin, Crossed products of semisimple cocommutative Hopf algebras, Proc. AMS 116 (1992), 321-327. MR 92m:16059

[4] W. Chin, D. Quinn, Rings graded by polycyclic-by-finite groups, Proc. AMS 102 (1988), 235-241. MR 89a:16001

[5] M. Cohen, D. Fischman, S. Montgomery, Hopf Galois extensions, smash products, and Morita equivalence, J. Algebra 133 (1990), 351-372. MR 91i:16068

[6] M. Cohen, S. Montgomery, Group-graded rings, smash products, and group actions, Trans. AMS 282 (1984), 237-258. MR 85i:16002

[7] M. Cohen, Ş. Raianu, S. Westreich, Semi-invariants for Hopf algebra actions, Israel J. Math. 88 (1994), 279-306. MR 95j:16042

[8] S. Dăscălescu, C. Năstăsescu, Ş. Raianu, Hopf Algebras: an Introduction, Pure and Applied Mathematics, A series of Monographs and Textbooks, vol. 235, Marcel Dekker Inc., New York-Basel, 2001. MR 2001j:16056

[9] J.W. Fisher, S. Montgomery, Semiprime skew group rings, J. Algebra 52 (1978), 241-247. MR 58:772

[10] T.Y. Lam, Lectures on Modules and Rings, GTM 189, Springer, 1999. MR 99i:16001

[11] B. Lemonnier, Dimension de Krull et dualité de Morita dans les extensions triangulaires, Comm. Algebra 12 (1984), 3071-3110. MR 86g:16034

[12] S. Montgomery, Hopf algebras and their actions on rings, CBMS Reg. Conf. Series 82, Providence, R.I., 1993. MR 94i:16019

[13] S. Montgomery, H.-J. Schneider, Prime ideals in Hopf Galois extensions, Israel J. Math. 112 (1999), 187-235. MR 2001e:16075

[14] C. Năstăsescu, F. Van Oystaeyen, Graded ring theory, Math. Library 82, North Holland, 1982. MR 84i: 16002

[15] D. Passman, It's essentially Maschke's theorem, Rocky Mountain J. Math. 13 (1983), 37-54. MR 84e:16023 
[16] D. Quinn, Group-graded rings and duality, Trans. AMS 292 (1985), 155-167. MR 87d:16002

[17] H.-J. Schneider, On inner actions of Hopf algebras and stabilizers of representations, J. Algebra 165 (1994), 138-163. MR 95d:16055

[18] E.A. Whelan, Finite subnormalizing extensions of rings, J. Algebra 101 (1986), 418-432. MR 88e:16042

Department of Mathematics, Syracuse University, Syracuse, New York 13244

E-mail address: dpquinn@syr.edu

Department of Mathematics, Syracuse University, Syracuse, New York 13244

E-mail address: sraianu@syr.edu

Current address: Department of Mathematics, California State University Dominguez Hills, 1000 E Victoria Street, Carson, California 90747

E-mail address: sraianu@csudh.edu 